\title{
EVALUATION OF A PROTOTYPE LOW-LEVEL LIQUID SCINTILLATION MULTISAMPLE COUNTER
}

\author{
SIGURDUR A. EINARSSON
}

Science Institute, University of Iceland, Dunhaga 3, 107 Reykjavík, Iceland

\begin{abstract}
A new, improved photomultiplier has been incorporated and a more efficient active guard installed in a single photomultiplier liquid scintillation counter. A special quartz vial was designed to improve the photoelectron cathode index (photoelectrons $/ \mathrm{keV}$ ), and thus, to reduce background. Four photomultipliers were installed so that four samples can be counted simultaneously. The measurements presented here cover background spectra and counting efficiency obtained under various conditions in the laboratory. These measurements and derived parameters, such as the figure of merit $\left(E^{2} / B\right)$ and the factor of merit $(S / \sqrt{ } B)$, are used to evaluate the system performance. Also presented are the results of measurements on the cathode index and further background spectra, obtained at an underground site.
\end{abstract}

\section{INTRODUCTION}

The present study is a continuation of the first phase of a study on the pulse spectrum of a simple, single photomultiplier liquid scintillation counter (Einarsson \& Theodórsson 1989). Several improvements have since been made to the system, based on the knowledge obtained from the original work and from the current study.

One of the conclusions reached in the radiocarbon intercomparison study, Stage 1, completed in September 1987 (Scott et al. 1989) is that the major component of variability in the results lies in the counting process. The authors also concluded that a systematic bias existed among laboratories. Polach (1989) suggested that some of the reasons for this bias may be that some laboratories do not test periodically (several times each year) freshly prepared, internationally accepted standard and background samples, and that vials and detectors are not individually calibrated. Polach suggests a program of interlaboratory comparison in which laboratories could obtain a set of unknown certified reference material samples to be determined. This major step towards quality assurance will help to maintain the high standard of dating with which liquid scintillation counting (LSC) should function. Frequent standard sample and background checks in LSC requires counting time. A compromise has to be struck between counting capacity and quality assurance, which is essentially achieved with the new low-level liquid scintillation multicounter (LSMC) presented here.

The LSMC is a new generation of liquid scintillation counters with radical design changes, capable of measuring four samples simultaneously. The system has many advantages: 1) longer counting times may be achieved while maintaining counting capacity, thus increasing accuracy; 2) while maintaining standard counting times, the counting capacity is improved as much as fourfold; 3 ) the four-sample system can also be used partly to check frequently counter stability with known, freshly-prepared international standards and an internationally accepted background standard continuously rotated among counting chambers; 4) quality assurance can be established through frequent determinations of Certified Reference Material samples (P.olach 1989).

Modern counters use two photomultiplier tubes (PMT) counting in coincidence to reduce background. The low-level LSMC is simpler in design. With maximum light collection, the ${ }^{14} \mathrm{C}$ pulses are brought over the tail of the spectrum of the dark counts, allowing low background. A single PMT for each sample ensures compact construction and an efficient $\mathrm{NaI}(\mathrm{Tl})$ guard counter that provides low background and immunity from fluctuations in cosmic radiation caused by barometric variations. 
In the last few years, the emphasis in counter development has been on reducing background to achieve the maximum obtainable age. If the background is less than ca. $20 \%$ of the sample count rate, its effect on counting statistics will be insignificant, provided the background is known to be stable. Under these conditions, the ultra-low background will neither improve the dating precision of relatively recent samples, nor increase the counting capacity for these samples. It is quite expensive to collect and prepare samples for counting. Samples often require equipment that can provide higher precision than most dating laboratories can afford. A multicounter system, such as the LSMC, can approach a solution to this problem.

\section{METHOD}

\section{Theory of Operation}

The conventional set-up of two opposing PMTs uses coincidence counting to help eliminate PM dark noise. New, low dark noise PMTs and good light collection have made it possible to use a single PMT in an LS system for low-level ${ }^{14} \mathrm{C}$ measurements.

The use of quartz has several advantages for this system: 1) it shows low background because of its low radioactive impurities: 2) its spectral transmission characteristics are excellent for scintillation light: 3) it is inert and does not have memory or absorb the scintillator cocktail.

Special attention to light collection is necessary with one PMT. Most of the light produced in an event should reach the cathode. This may be accomplished with a reflector arrangement. The diffuse reflecting property of Teflon makes it an ideal reflecting material in the spectral range of scintillation light ( $c a .400 \mathrm{~nm}$ ). Reflector surfaces, such as aluminum, are excellent mirror reflectors, but oxidation on surfaces reduces reflection properties, and repeated reflections from wall to wall increase the probability of light absorption.

Cosmic radiation is a major source of background in LSC. Muon contribution in background can be removed easily, but secondary $\gamma$ radiation produced by muons and protons is more difficult to eliminate. The contribution of secondary $\gamma$-rays can be practically eliminated (Theodórsson \& Heuser 1991) using a guard counter with high $\gamma$-efficiency. A large $\mathrm{NaI}(\mathrm{Tl})$ has $100 \%$ muon efficiency, close to $100 \%$ intrinsic $\gamma$ detection efficiency below $500 \mathrm{keV}$ and high efficiency for higher energies.

\section{INSTRUMENTATION}

\section{Experimental System}

We constructed a new, experimental LSC with a single PMT (Fig. 1) based on earlier results obtained by Einarsson and Theodórsson (1989). We also used a more efficient $\mathrm{NaI}(\mathrm{Tl}) 10-\mathrm{cm}$ wide well crystal guard counter to minimize background from cosmic radiation. We made a special quartz vial to minimize loss of light and background from vial radioimpurities. Three or more layers of Teflon tape provide diffused reflection and, at the same time, secure the vial to the PMT. The detectors are shielded with $20 \mathrm{~cm}$ of iron against local radioactivity. We use standard counting electronics to amplify the pulses, and a stable, low-noise, high-voltage power supply ensures consistent performance. The pulse-height analyzer measures both coincidence and anticoincidence spectra.

\section{Prototype Multisample LSC}

Figure 2 shows the prototype multicounter system. Four PMTs are arranged in a tight matrix to fit into the well of a $15 \mathrm{~cm} \mathrm{NaI(Tl)}$ guard counter. Sample vials are arranged in a sample tray, 


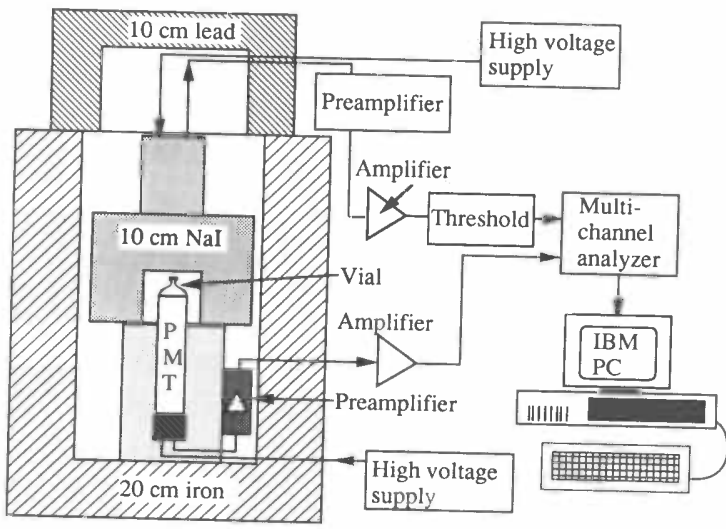

Fig. 1. Schematic diagram showing the basic construction of the experimental liquid scintillation counter

which is placed exactly above the PMTs. The assembly is then lifted into the NaI(Tl) well and enclosed in $10-\mathrm{cm}$-thick lead housing. The dimensions of the prototype LSMC are $\mathrm{ca} .30 \times 30 \times$
$100 \mathrm{~cm}$.

Each PMT and vial with electronic circuitry constitutes a counting channel. Each channel has an individual high-voltage supply, pulse-processing electronics and multichannel analyzer. The ana-

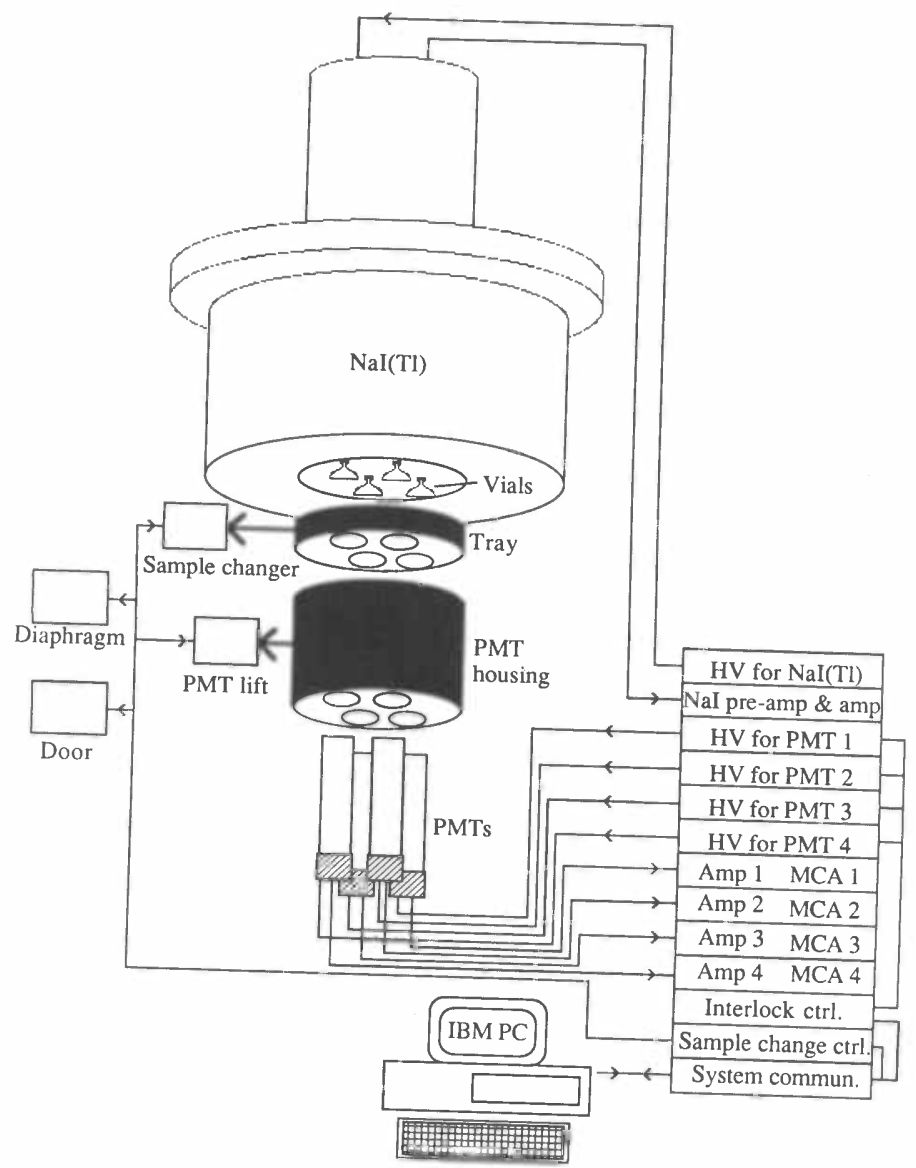

Fig. 2. Main parts of the LSMC system 
lyzers are computer-controlled and data are transferred to the computer for display and processing. The only pulse processing done is that of pulse-height analysis.

The PMTs are specially selected for low background. The tube is made of low-K glass, which helps to reduce dark noise. Cerenkov pulses in the window are minimized by making the window very thin. The windows have been sandblasted to ensure maximum light collection efficiency.

Samples are changed under low-intensity red light and with the high-voltage supply switched off. Under these controlled light conditions, no fluorescence problems were observed.

\section{MEASUREMENTS}

Measurements were made on both the experimental LSC and the prototype LSMC. In both cases, a known amount of background sample or ${ }^{14} \mathrm{C}$ sample, prepared in benzene with $1.7 \%$ butyl PBD scintillant, is placed in the counting vial. An ${ }^{241} \mathrm{Am}$ external standard (60 $\mathrm{keV} \gamma$-rays) is used for energy calibration and to check system stability during the counting period.

The present method of manufacture produces small variations in the volume of the vials, ranging from 2.6 to $3.8 \mathrm{ml}$, although the general vial shape and other characteristics are the same. In the experimental system, the vial is placed over the PMT in contact with the window. About three layers of Teflon tape are wrapped around the vial and the sides of the PMT to anchor the vial. In the LSMC, three or more layers of Teflon tape are wrapped around the vials, which are then placed in the tray. Once the tray is in place above the PMTs, the two systems exhibit similar lightcollection characteristics.

To measure the relative light-collection efficiency of an LSC, it is necessary to introduce the cathode index, $\eta$ (Einarsson \& Theodórsson 1989) given by

$$
\eta=S_{x} \cdot G \cdot C_{p l} / E_{a}
$$

where $S_{x}$ is the scintillation efficiency, $G$ is light-collection efficiency, $C_{p t}$ is the phototube response factor and $E_{a}$ is the energy absorbed in the scintillator. $\eta$ measures the number of electrons released by a scintillator light pulse at the cathode per $\mathrm{keV}$ deposited in the scintillator by the charged particle. As $\eta$ increases, the contribution of the photomultiplier dark noise to the background decreases. This is an important parameter for system calibration. To measure $\eta$, the one photoelectron equivalent is established followed by an energy calibration with a $60 \mathrm{keV}{ }^{241} \mathrm{Am}$ external $\gamma$-source.

The efficiency of the $\mathrm{NaI}(\mathrm{Tl})$ detector to remove the cosmic contribution from the background is established with measurements on the experimental system $18 \mathrm{~m}$ below ground in a hydroelectric power plant in southwest Iceland. This is equivalent to an absorbing layer of $c a .48 \mathrm{~m}$ of water, which reduces the muon flux by a factor of 15 (George 1957). To ensure similar environments above and below ground, the experiment was performed in the same passive guard made of $15 \mathrm{~cm}$ iron and $10 \mathrm{~cm}$ lead surrounding the counting chamber.

\section{RESULTS AND DISCUSSION}

Table 1 presents the cathode index for the experimental system and the LSMC. Considerable variation exists in the index value between counter elements, indicating different light-collection efficiencies. The low index in Channel 1 results from a drop in light-collection efficiency due to the sandblasted surface of the vial. Other variances can be attributed to differences in Teflon reflector thickness, slight variations in the vial shape and differences in the PMT response factor. 
TABLE 1. Vial volume, cathode index, $\eta$ and performance of the experimental system and the LSMC for the measurement of ${ }^{14} \mathrm{C}$ at $71 \%$ counting efficiency. The maximum obtainable age figures, $\mathrm{T} 1_{\max }$ and $\mathrm{T} 2_{\max }$, are calculated with 3 and $9 \mathrm{k}$ min counts, respectively.

\begin{tabular}{lcccccccc}
\hline $\begin{array}{c}\text { System/ } \\
\text { channel no. }\end{array}$ & $\begin{array}{c}\text { Vial volume } \\
(\mathrm{ml})\end{array}$ & $\begin{array}{c}\eta \\
\mathrm{e} / \mathrm{keV}\end{array}$ & $\begin{array}{c}\mathrm{B} \\
(\mathrm{cpm})\end{array}$ & $\begin{array}{c}\mathrm{S}^{*} \\
(\mathrm{cpm})\end{array}$ & $\mathrm{E}^{2} / \mathrm{B}$ & $\mathrm{S} / \sqrt{ } \mathrm{B}$ & $\begin{array}{c}\mathrm{T} 1_{\max } \\
(\mathrm{ka})\end{array}$ & $\begin{array}{c}\mathrm{T}_{\max } \\
(\mathrm{ka})\end{array}$ \\
\hline Experimental & 3.2 & 1.57 & 0.32 & 24.99 & 15753 & 44.18 & 54.2 & 58.7 \\
LSMC 1 & 3.3 & 1.23 & 0.56 & 25.77 & 9002 & 34.44 & 52.2 & 56.7 \\
LSMC 2 & 3.5 & 1.69 & 0.42 & 27.34 & 12002 & 42.19 & 53.9 & 58.3 \\
LSMC 3 & 3.8 & 1.67 & 0.31 & 29.68 & 16261 & 53.31 & 55.7 & 60.2 \\
LSMC 4 & 2.6 & 1.45 & 0.45 & 20.31 & 11202 & 30.28 & 51.2 & 55.6 \\
\hline
\end{tabular}

*Count rate converted to $95 \%$ oxalic acid standard

Figure 3 shows the variation in background with energy interval (window). The window limits of $16,20,25,30$ and $35 \mathrm{keV}$ correspond, respectively, to ca. 81, 77, 71, 66 and $61 \%$ counting efficiency. We determined the counting efficiency in the experimental system and in the four channels in the LSMC using a known local standard with an activity of $c a .83 \mathrm{dpm} \mathrm{g}^{-1}$. We also determined the counting efficiencies of Channels 1,2 and 3 using the measured counting efficiency

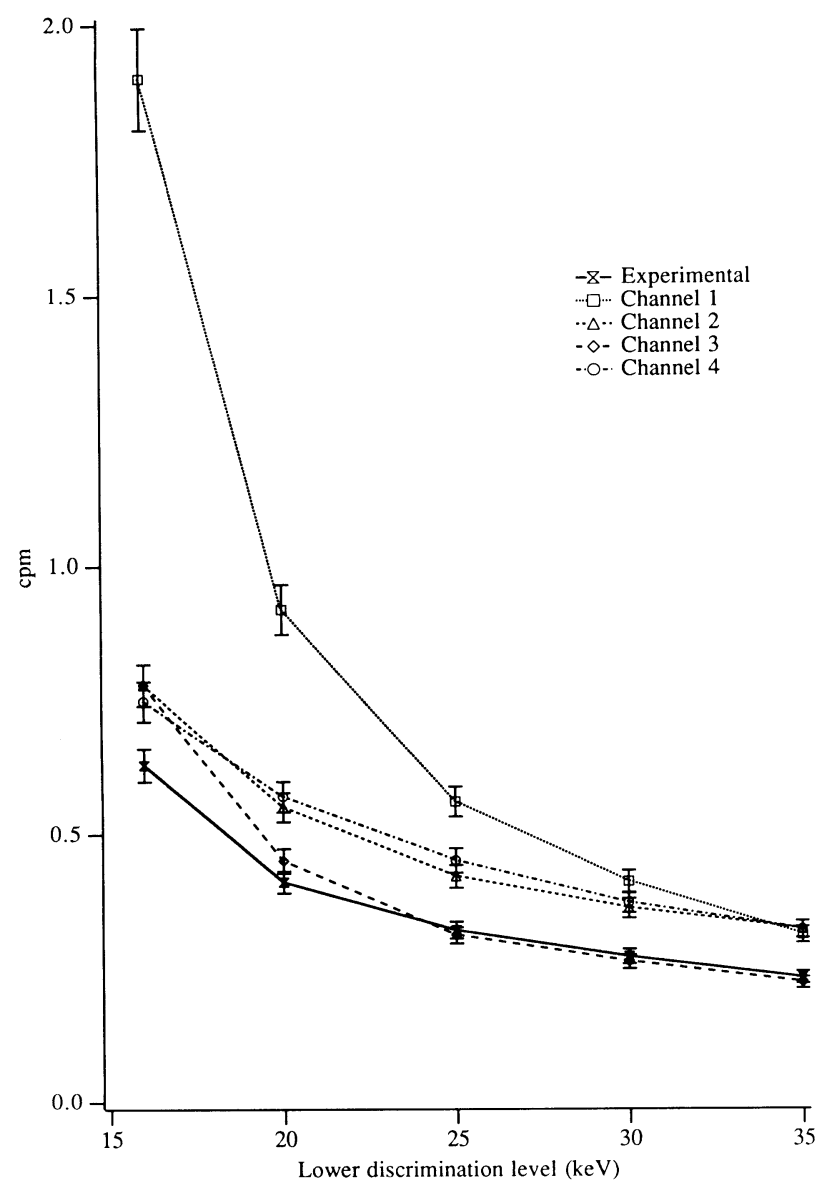

Fig. 3. The background in different windows of the experimental and prototype systems as a function of lower threshold energy. Each curve represents a counting channel in the LSMC or in the experimental system, according to the key. 
of Channel 4 and the ${ }^{241} \mathrm{Am}$ external standard. The relatively high background in Channel 1, particularly at high counting efficiencies, is explained by the reduction in light collection efficiency reflected in the low cathode index. Here, the ${ }^{14} \mathrm{C}$ spectrum is shifted towards lower channels in the pulse-height analyzer, overlapping a larger fraction of the photomultiplier dark noise spectrum. As counting efficiency drops, the difference becomes less pronounced.

Channel 3 and the experimental system exhibit the lowest overall background. Channels 2 and 4 show similar, slightly poorer characteristics but with minor differences. This encouraging result establishes a firm basis for continued development of the LSMC.

A rough check on the system shows comparable activity measurements on a local standard treated as an unknown: $13.0 \pm 0.2 \mathrm{dpm} \mathrm{g}^{-1}$ in the LSMC and $13.2 \pm 0.2 \mathrm{dpm} \mathrm{g}^{-1}$ in the experimental system. The results agree with the local standard activity of $13.6 \pm 0.7 \mathrm{dpm} \mathrm{g}^{-1}$.

The backgrounds in Channels 1, 2 and 3 were measured, whereas the known local standard was measured in Channel 4. No appreciable difference was observed between the background values with or without the sample in Channel 4. This shows that cross-talk among channels is within acceptable limits.

Figure 4 shows the spectrum of the unknown ${ }^{14} \mathrm{C}$ standard in Channel 4 . The rapid increase in the slope below $15 \mathrm{keV}$ is mostly due to photomultiplier dark noise.

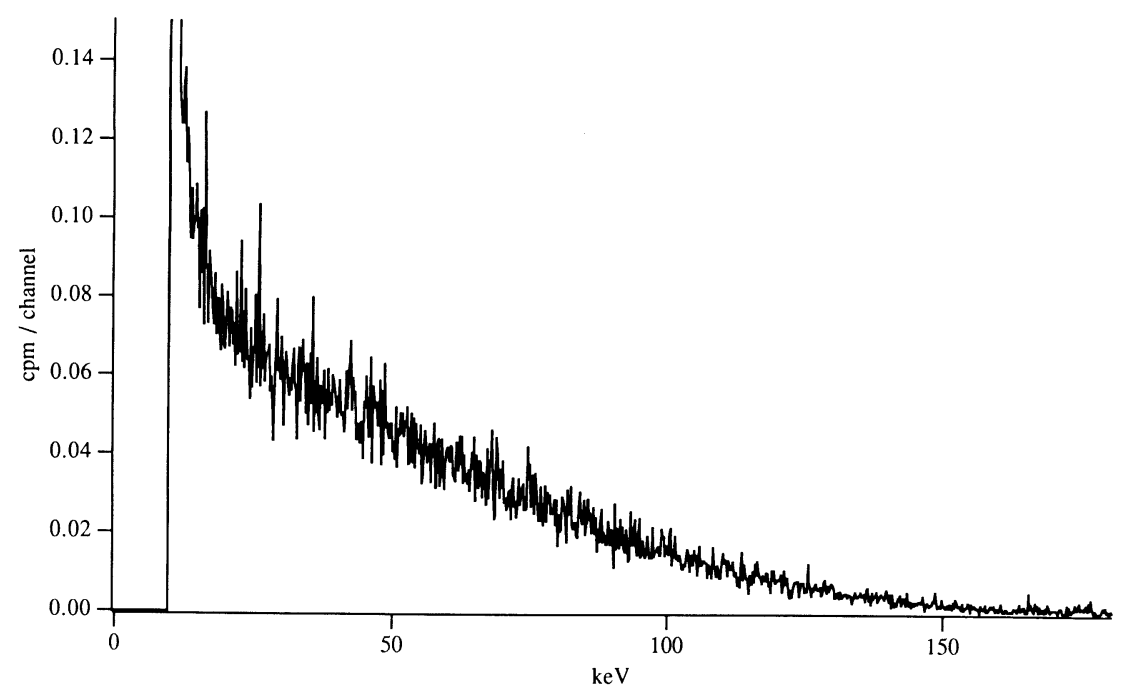

Fig. $4 .{ }^{14} \mathrm{C}$ spectrum in Channel 4 of the LSMC

The performance of the experimental and LSMC counters is evaluated for a counting efficiency of ca. $71 \%$. Results appear in Table 1 . The figure of merit, $\mathrm{E}^{2} / \mathrm{B}$, is calculated together with the factor of merit, $\mathrm{S} / \sqrt{ } \mathrm{B}$ ( $\mathrm{S}$ is the net count rate of $95 \%$ oxalic acid standard). With increased counting capacity, longer counting times are possible, resulting in higher precision. To get an idea of the improvement in precision with increased counting time, the maximum obtainable age is calculated for 3 and $9 \mathrm{k}$ min counts using

$$
\mathrm{T}_{\max }=8033(\ln (\sqrt{ }(\mathrm{T} / 8))+\ln (\mathrm{S} / \sqrt{ } \mathrm{B}))
$$

which is derived using the $2 \sigma$ criterion with $T$ as the counting time in minutes (Gupta \& Polach 
1985). The best performance is obtained in Channel 3, where a relatively large sample volume and low background combine to give a maximum obtainable age of $55.7 \mathrm{ka}$ after $3 \mathrm{kmin}$. It is interesting to compare this performance with commercial counters. Table 2 shows how the performances of different counters compare (Polach et al. 1988).

TABLE 2. Comparison of performance between commercial counters and the LSMC. Counting time is $3 \mathrm{k} \mathrm{min}$.

\begin{tabular}{llllll}
\hline System & $\begin{array}{c}\text { Vial } \\
\text { material }\end{array}$ & $\begin{array}{c}\text { Volume } \\
(\mathrm{ml})\end{array}$ & $\begin{array}{c}\mathrm{B} \\
(\mathrm{cpm})\end{array}$ & $\mathrm{E} \%$ & $\begin{array}{c}\mathrm{T}_{\max } \\
(\mathrm{ka})\end{array}$ \\
\hline LSMC & Quartz & 3.8 & 0.31 & 71 & 55.7 \\
Packard 3330* & Teflon & 3 & 0.31 & 72.9 & 54.1 \\
Quantulus & Teflon/Cu & 3 & 0.21 & 76.4 & 56.0 \\
Quantulus** & Teflon/Cu & 3 & 0.14 & 70 & 57.0 \\
Packard 2250 & ${ }^{40} \mathrm{~K}$ free & 3.5 & 0.43 & 64 & 53.0 \\
\hline
\end{tabular}

*Underground, Warragamba dam wall, very high cosmic shielding (Polach et al. 1988)

**Surface lab in cosmic-ray shielded and $\gamma$-ray-free environments (Polach et al. 1988)

${ }^{\dagger}$ Converted (burst counting) (Noakes \& Valenta 1989)

Measurements in the experimental system in the underground site at Írafoss confirm the excellent efficiency of the $\mathrm{NaI}(\mathrm{Tl})$ crystal. The passive shielding for the experimental system in this comparison study is thinner than during normal measurements and a different vial is in use. Background at the surface site gives $0.46 \pm 0.02 \mathrm{cpm}$ in a $71 \%$ counting window, whereas the background at the underground site is $0.45 \pm 0.01 \mathrm{cpm}$. The coincidences occurring in the highest channel of the pulse-height analyzer correspond to mesons passing through both vial and $\mathrm{NaI}(\mathrm{Tl})$. Surface coincidences measured $4.04 \mathrm{cpm}$, whereas at the underground site, the coincidences were $0.56 \mathrm{cpm}$. This corresponds to a reduction by a factor of 7.2 .

\section{CONCLUSION}

This study has shown low background levels can be obtained with a single PMT in conjunction with an effective $\mathrm{NaI}(\mathrm{Tl})$ guard counter. A single PMT, instead of two opposing PMTs, permits a compact construction of several detectors mounted in the same unit. Our multicounter system was constructed on the basis of this principle.

Distinct advantages may be gained with LSMC systems. Through increased counting capacity, running quality assurance programs becomes easier. The system size is small because of the way the four PMTs are arranged. Comparison with modern, commercial low-level LSCs show them to be an attractive alternative.

\section{ACKNOWLEDGMENTS}

I wish to thank Páll Theodórsson, who proposed the original idea for this study, for his invaluable advice and constant encouragement during this study and for his critical review of this manuscript. 


\section{REFERENCES}

Einarsson, S. A. and Theodórsson, P. 1989 Study of background pulse spectrum of an LSC system. In Long, A. and $\mathrm{Kra}, \mathrm{R}$. S., eds., Proceedings of the 13th International ${ }^{14} \mathrm{C}$ Conference. Radiocarbon 31 (3): 342-351.

George, E. P. 1957 Observations of cosmic rays underground and their interpretation. In Wilson, J. G., ed., Progress in Cosmic Ray Physics 1: 395-416.

Gupta, K. G. and Polach, H. A. 1985 Radiocarbon Dating Practices at ANU, Handbook. Radiocarbon Laboratory, Research School of Pacific Studies, ANU, Canberra: 94-99.

Noakes, J. E. and Valenta, R. J. 1989 Low background liquid scintillation counting using an active sample holder and pulse discrimination electronics. In Long, A. and Kra, R. S., eds., Proceedings of the 13th International ${ }^{14} \mathrm{C}$ Conference. Radiocarbon 31(3): 332341.
Polach, H. $1989{ }^{14}$ CARE. In Long, A. and Kra, R. S., eds., Proceedings of the 13th International ${ }^{14} \mathrm{C}$ Conference. Radiocarbon 31(3): 422-428.

Polach, H., Calf, G., Harkness, D., Hogg, A., Kaihola, L. and Robertson, S. 1988 Performance of new technology liquid scintillation counters for ${ }^{14} \mathrm{C}$ dating. Preprint. Nuclear Geophysics 2(2).

Scott, E. M., Aitchison, T. V., Harkness, D. D., Baxter, M. S. and Cook, G. T. 1989 An interim progress report on Stages 1 and 2 of the international collaborative program. In Long, A. and $\mathrm{Kra}, \mathrm{R}$. S., eds., Proceedings of the 13 th International ${ }^{14} \mathrm{C}$ Conference. Radiocarbon 31(3): 414-421.

Theodórsson, P. and Heuser, G. 1991 External guard counters for low-level counting systems. Nuclear Instruments and Methods B53: 97-100. 